\title{
C-03
}

\section{MODELO HIDRÁULICO PARA LA OPTIMIZACIÓN DE SUBUNIDADES IRREGULARES DE RIEGO POR GOTEO}

\author{
Cervera Gascó, $\mathrm{J}^{1}$, Del Castillo Sánchez-Cañamares, $A^{2}$, Montero Martinez, $\mathrm{J}^{3}$, Tarjuelo \\ Martín-Benito, J.M. ${ }^{4}$, Moreno Hidalgo, M.A ${ }^{5}$
}

${ }^{1}$ Ingeniero Agrónomo, Contratado predoctoral; Centro Regional de Estudios del Agua (CREA), UCLM, Ctra de Las Peñas km. 3.2, 02071 Albacete, Jorge.Cervera@uclm.es

${ }^{2}$ Ingeniero Técnico Agrícola, Investigador; Centro Regional de Estudios del Agua (CREA), UCLM, Ctra. de Las Peñas km. 3.2, 02071 Albacete; Amaro.Castillo@uclm.es

${ }^{3}$ Dr. Ingeniero Agrónomo, Titular de universidad; Centro Regional de Estudios del Agua (CREA), UCLM, Ctra de Las Peñas km. 3.2, 02071 Albacete; Jesus.Montero@uclm.es

${ }^{4}$ Dr. Ingeniero Agrónomo, Catedrático de Universidad; Centro Regional de Estudios del Agua (CREA), UCLM, Ctra de Las Peñas km. 3.2, 02071 Albacete; jose.tarjuelo@uclm.es

${ }^{5}$ Dr. Ingeniero Agrónomo, Profesor Contratado Doctor; Centro Regional de Estudios del Agua (CREA), UCLM, Ctra de Las Peñas km. 3.2, 02071 Albacete; MiguelAngel.Moreno@uclm.es

\section{Resumen}

La escasez de los recursos hídricos actuales, especialmente en las zonas con clima árido o semiárido, provocan la necesidad de desarrollar herramientas de ayuda en la toma de decisiones para el diseño y manejo del riego, y más cuando se pretende utilizar energía fotovoltaica. El objetivo del trabajo ha sido desarrollar un modelo hidráulico que pueda acoplarse al modelo fotovoltaico, para el caso más general de subunidades de riego de forma y topografía irregular, constituyendo una herramienta de ayuda en la toma de decisiones para el diseño y manejo del riego con energía fotovoltaica, analizando la calidad del riego en las distintas subunidades con forma y topografía irregular. Para la calibración y validación del modelo, se ha aplicado a una parcela de 90 ha, de un cultivo de almendro regado por goteo, situada en una zona con topografía y forma muy irregular. Los resultados permiten identificar las zonas con problemas en la uniformidad de emisión en función de la presión disponible en la entrada de la subunidad de riego.

\section{Abstract}

Nowadays, water resources are limited especially in places with arid and semi-arid conditions. For that reason, it is essential the development of decision support system models (DSS) aiming the design and management of irrigation systems, especially when they are fed with photovoltaic energy. The main objective of this study was the development of a hydraulic model linked with a photovoltaic model, considering the most general case of irrigation subunits with irregular shape and topography. Thus, a decision support system tool (DSS) for the design and management irrigation with photovoltaic power is developed.

Analyzing the irrigation quality in different subunit with irregular shape and topography is a main issue in precision irrigation. For model calibration and validation has been applied in an almond field with 90 ha with drip irrigation located in a shape and topography land. The results enabled us to identify the areas with uniformity problems depending on the pressure in the subnit head. 


\section{1- Introducción y Objetivos}

Tal y como indica Daccache et al (2014) la agricultura sostenible debería ser respaldada por políticas compensatorias entre el ahorro de agua, las emisiones de CO2 y la producción de alimentos, particularmente en las regiones con escasez de agua como ocurre en la cuenca mediterránea, donde debido a la intensificación de la agricultura, las necesidades de bombeo son aún mayores al tener que utilizar en gran medida aguas subterráneas

No obstante, el óptimo de funcionamiento de un sistema de riego, desde un punto de vista ecológico, solamente se podrá conseguir considerando simultáneamente criterios medioambientales y económicos (Carrillo-Cobo et al., 2014).

En el diseño de las diferentes subunidades de riego hay que tener en cuenta, la diferente cota de los emisores, provocada por la irregularidad del terreno, las pérdidas de carga en la tubería y las características propias del emisor en cuanto a su coeficiente de variación de fabricación, exponente de descarga, sección de paso, sistema de filtrado, etc., lo que condiciona la uniformidad de emisión y la eficiencia de riego, siendo estos indicadores fundamentales para caracterizar la calidad del riego.

El desarrollo de herramientas y modelos, de ayuda en la toma de decisiones para el diseño y el manejo de las instalaciones de riego contribuye al ahorro de agua y energía, tanto en instalaciones nuevas como en las que están en funcionamiento Así, Carrion et al. (2013 y 2014) desarrollaron la herramienta PRESUD para el diseño óptimo de subunidades regulares de riego a presión, tanto localizado como aspersión, buscando el coste total mínimo (operación más inversión) por unidad de superficie regada, teniendo en cuenta la uniformidad de emisión y la eficiencia de aplicación en las subunidades de riego A su vez, Moreno et al. (2016) han desarrollado la herramienta PRESUD-irregular, que permite el diseño de mínimo coste total de subunidades de riego a presión en parcela con forma y topografía irregulares.

Carrion et al (2016) desarrollaron una herramienta llamada DOPIR (Dimensioning Of Pressurized IRrigation) para la optimización del proceso de extracción y aplicación del agua con el sistema de riego, buscando el coste total mínimo (inversión más operación), contemplando el proceso en su conjunto, desde la fuente de agua hasta el emisor.

El objetivo del trabajo es desarrollar un modelo hidráulico que pueda acoplarse a un modelo fotovoltaico, previamente desarrollado, para analizar la calidad del riego en las distintas subunidades de forma y topografía irregular en que se haya dividido la parcela a regar, que se alimentan directamente de un depósito elevado o de una bomba alimentada con energía fotovoltaica. Para la calibración y validación del modelo, se aplica a una parcela de 90 ha, de un cultivo de almendro regado por goteo, situada en una zona con topografía y forma muy irregular.

\section{2- Materiales y métodos}

Para la realización de este trabajo se ha utilizado una parcela de 90 ha de almendros, con marco de $7 \times 7 \mathrm{~m}^{2}$ regada por goteo La finca está situada en el término municipal de Hellín, al sureste de la provincia de Albacete, y está dividida en 18 subunidades, que están ubicadas en una topografía muy irregular, con grandes diferencias de pendiente incluso dentro de la misma subunidad.

Los goteros utilizados son autocompensantes, con un caudal de diseño de $2 \mathrm{l} / \mathrm{h}$, habiéndolos ensayado en laboratorio para obtener su curva de descarga y el coeficiente de 
variación de fabricación. El agua suministrada a la plantación procede de un sondeo con nivel dinámico situado a $200 \mathrm{~m}$, utilizando una bomba sumergida de $26 \mathrm{~kW}$ alimentada con energía fotovoltaica. Además, la finca cuenta con un embalse de $12.500 \mathrm{~m}^{3}$ aproximadamente.

En la figura 1 se muestra el plano con las subunidades, la red de distribución, ubicación de las placas solares, pozo y embalse.

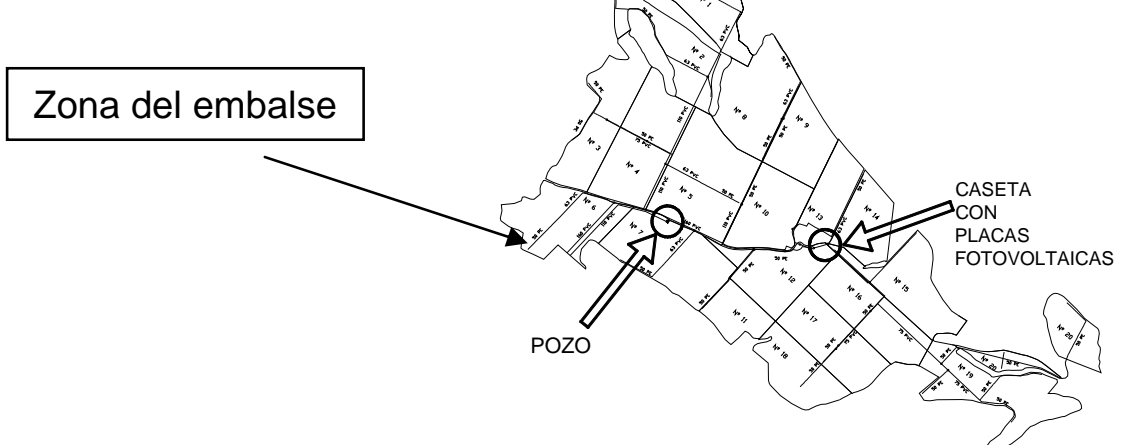

Figura 1. Plano de la finca con las subunidades, la red de distribución, ubicación de las placas solares, pozo y embalse.

En la cubierta de la caseta, que se encuentra en la parte central de la finca, están instaladas 136 placas solares, que aportan una potencia máxima de $30 \mathrm{~kW}$, con la que se alimenta la bomba través de un variador de velocidad.

Con el fin de tener una precisa caracterización de la finca, se ha realizado un levantamiento topográfico con GPS-RTK, y precisión centimétrica en altimetría, identificando además el inicio y fin de cada ramal, y la localización de cada una de las válvulas de apertura de las subunidades, consiguiendo así una muy buena caracterización del sistema de riego instalado. En la figura 2 se muestra una ortofoto con todos los puntos obtenidos con GPS-RTK.



Figura 2. Medición de puntos de inicio y final de ramal así como de las válvulas de apertura de cada subunidad mediante GPS-RTK.

Además, se han realizado diferentes vuelos mediante vehículos aéreos no tripulados (drones) utilizando un drone de tipo cuadracóptero md-400 con el fin de obtener el modelo digital del terreno (MDT), lo que ha permitido determinar la cota de cada uno de los goteros con precisión centimétrica.

La información obtenida ha permitido reproducir el diseño hidráulico de la red de distribución y de las distintas subunidades de riego. Para la obtención del modelo hidráulico 
de la instalación se ha utilizado el motor de cálculo EPANET (Rossman, 2000), implementado en un software propio desarrollado en Matlab®.

La descarga de los goteros se ha estimado con la ecuación (Karmeli y Keller, 1975), obtenida en laboratorio:

$$
\mathrm{q}_{\mathrm{h}}=\mathrm{K} \cdot \mathrm{h}_{\mathrm{e}}^{\mathrm{x}}
$$

Siendo $q_{\mathrm{h}}$ el caudal del emisor, $\mathrm{K}$ el coeficiente del emisor, $\mathrm{x}$ el exponente del emisor y $h_{e}$ la presión en cabecera del emisor.

Con ayuda de la herramienta PRESUD-irregular se ha deducido la ecuación de descarga de cada subunidad, que determina el caudal descargado por el conjunto de goteros en función de la presión a la entrada de la subunidad, así como la presión y caudal descargado por cada uno de los goteros. Esto permite calcular los correspondientes coeficientes de uniformidad de emisión, y los coeficientes de variación de caudal y presión en cada una de las subunidades, teniendo una muy buena caracterización de la calidad del riego.

La simulación hidráulica de la red de distribución figura 3 permite establecer la presión en cada uno de los nudos o hidrantes a partir de la presión y caudal en cabecera, para cualquier combinación de subunidades de riego abiertas.

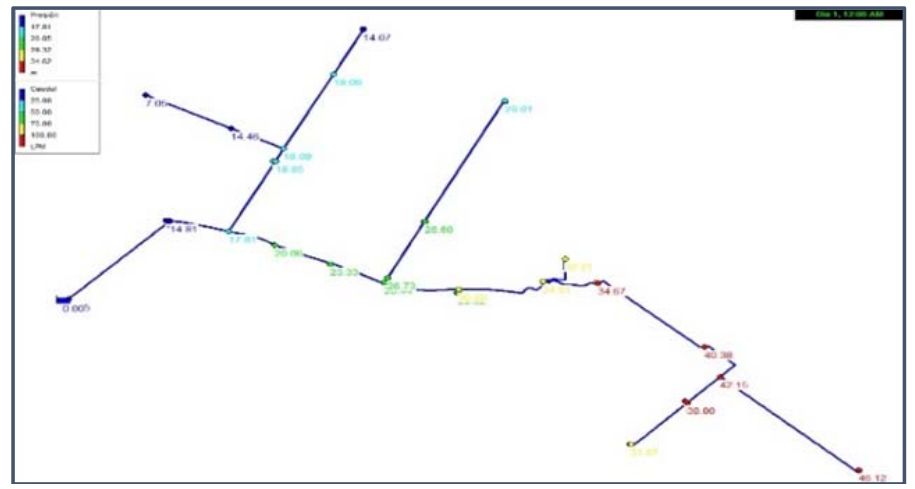

Figura 3. Simulación de la red de distribución con presión en cada uno de los nudos.

El modelo hidráulico de la red y de las subunidades de riego permite identificar las subunidades que podían ser regadas adecuadamente desde el embalse, situado en la parte más alta de la finca, así como establecer el valor de presión adicional que sería necesario tener en el origen de la red para obtener un mínimo valor de uniformidad de emisión en cada subunidad de riego.

\section{3- Resultados y discusión}

La herramienta generada que se ha desarrollado en Matlab® permite obtener los siguientes resultados. A modo de ejemplo, en la figura 4 se muestra la distribución de caudales de los goteros de una subunidad determinada asociada a una presión en cabecera de 45, 32, 19, y 7 m.c.a. En la figura 5 se muestra la distribución de caudales de los goteros de otra subunidad con forma muy distinta a la anterior para una presión en cabecera de 45 , 39, 33, 27 m.c.a. 
El rango de valores de presión en cabecera disponible es más grande pero en este caso se han seleccionado 4 valores de presión en cabecera representativos de cada subunidad.

a)

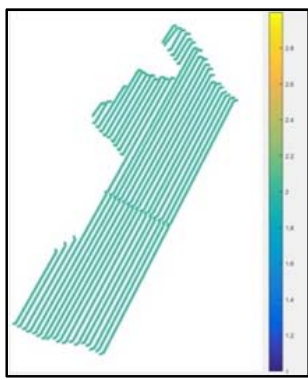

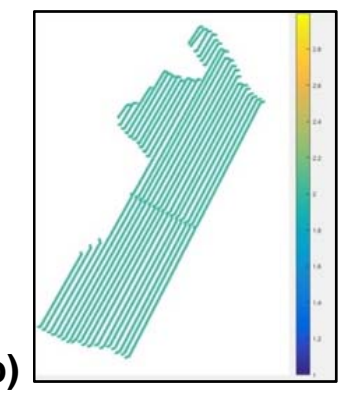

c)


Figura 4. Distribución de caudales de los goteros de una subunidad determinada asociada a una presión de 45 (a), 32 (b), 19 (c) y 7 m.c.a. de presión en cabecera dada.

En las figuras 4 a) y 4 b), se puede ver que la simulación indica que para una presión en cabecera de 45 m.c.a y 32 m.c.a todos los goteros esta autocompensando, es decir todos los goteros están suministrando el caudal de diseño que es de $2 \mathrm{l} / \mathrm{h}$. y por tanto la uniformidad de emisión es del $100 \%$.

En la figura $4 \mathrm{c}$ ), se puede ver que la simulación indica que para una presión en cabecera de 19 m.c.a el caudal máximo producido es de $2 \mathrm{l} / \mathrm{h}$ mientras que hay emisores que presentan un caudal mínimo de $1.66 \mathrm{l} / \mathrm{h}$. La diferencia de caudales es de un $17 \%$ y la uniformidad de emisión es del $98 \%$.

En la figura 4 d), se puede ver que la simulación indica que para una presión en cabecera de 7 m.c.a el caudal máximo producido es de $1.78 \mathrm{l} / \mathrm{h}$ mientras que hay emisores que presentan un caudal mínimo de $0.17 \mathrm{l} / \mathrm{h}$. La diferencia de caudales es de un $127 \%$ y la uniformidad de emisión es del $84 \%$.

a)

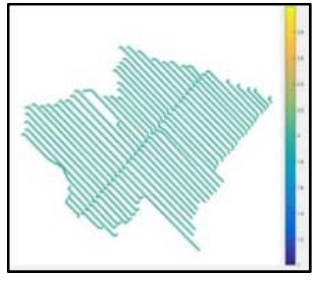

b)

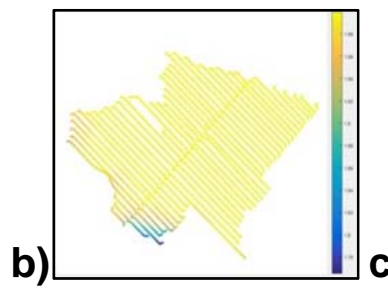

c)

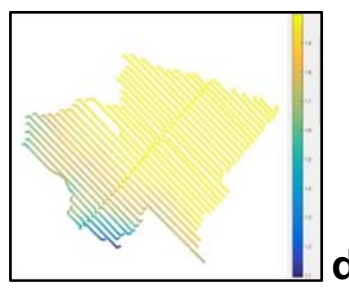

d)

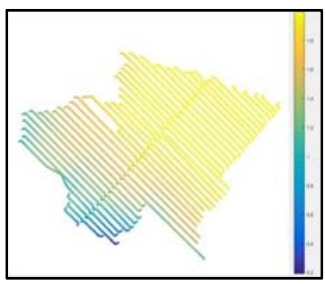

Figura 5. Distribución de caudales de los goteros de una subunidad determinada asociada a una presión de 45 (a), 39 (b), 33 (c) y 27 m.c.a. de presión en cabecera dada.

En la figura 5 a), podemos ver que la simulación indica que para una presión en cabecera de 45 m.c.a todos los goteros esta autocompensando, es decir todos los goteros están suministrando el caudal de diseño que es de $2 \mathrm{l} / \mathrm{h}$ y por tanto la uniformidad de emisión es del $100 \%$.

En la figura 5 b), podemos ver que la simulación indica que para una presión en cabecera de 39 m.c.a el caudal máximo producido es de $2 \mathrm{l} / \mathrm{h}$ mientras que hay emisores que presentan un caudal mínimo de 1,76 I/h. La diferencia de caudales es de un 11,77 \% y la uniformidad de emisión es del $99 \%$.

En la figura $5 \mathrm{c}$ ), podemos ver que la simulación indica que para una presión en cabecera de 33 m.c.a el caudal máximo producido es de $2 \mathrm{l} / \mathrm{h}$ mientras que hay emisores 
que presentan un caudal mínimo de 1,09 I/h. La diferencia de caudales es de un $47.52 \%$ y la uniformidad de emisión es del $94 \%$.

En la figura $5 \mathrm{~d}$ ), podemos ver que la simulación indica que para una presión en cabecera de 27 m.c.a. el caudal máximo producido es de $2 \mathrm{l} / \mathrm{h}$ mientras que hay emisores que presentan un caudal mínimo de 0,19 l/h. La diferencia de caudales es de un $106 \%$ y la uniformidad de emisión es del $82 \%$.

Como se puede ver, con los datos presentados, según la presión en cabecera de la subunidad, existen zonas cuyos emisores están aportando caudales muy bajos mientras que en otras zonas existen emisores que están trabajando con valores similares o iguales al caudal de diseño.

\section{4- Conclusiones y recomendaciones}

La irregularidad de las subunidades así como las grandes pendientes del terreno tienen una clara influencia sobre la presión en el origen de la subunidad y en consecuencia sobre el caudal y la uniformidad de emisión de la misma.

Los resultados obtenidos permiten conocer las curvas caudal-presión de cada subunidad, además de la uniformidad de emisión y el coeficiente de variación de presiones y caudales, entre otros, lo que permite cuantificar la calidad del riego, además de ayudar a establecer la secuencia optima de apertura de subunidades de acuerdo a la presión disponible en cabecera de la red de distribución. Esto, también permite identificar las zonas de la subunidad de riego con mayores problemas de falta de presión y caudal, lo que resulta fundamental para intentar buscar soluciones con distintos diámetros de tuberías, tipos de goteros, etc.

Queda clara pues la utilidad de la herramienta generada, y que el conocimiento de las necesidades de presión, caudal y uniformidad de emisión son fundamentales para decidir el momento óptimo del día para el riego según la energía generada por el sistema fotovoltaico, constituyendo una herramienta de gran ayuda para el diseño y dimensionamiento de las instalaciones con bombeo solar, dependiendo de la radiación solar disponible.

\section{5- Bibliografía}

Daccache, A., Ciurana, J.S., Rodriguez Diaz, J.A., Knox, J.W. (2014). Water and energy footprint of irrigated agriculture in the Mediterranean region. Environ. Res. Lett., 9, 124014 (12pp).

Carrillo-Cobo, M.T., Camacho Poyato, E., Montesinos, P., Rodríguez Díaz, J.A. (2014). Assessing the potential of solar energy in pressurized irrigation networks. The case of Bembézar MI irrigation district (Spain). Span. J. Agric. Res. 12 (3), 838-849.

Carrión, F., Montero, J., Tarjuelo, J.M., Moreno, M.A. (2014). Design of sprinkler irrigation subunit of minimum cost with proper operation. Application at corn crop in Spain. Water Resour. Manag., 28, 5073-5089.

Carrión, F., Sanchez-Vizcaino, J., Corcoles, J.I., Tarjuelo, J.M., Moreno, M.A. (2016). Optimization of groundwater abstraction system and distribution pipe in pressurized irrigation systems for minimum cost. Irrig Sci., 34, 145-159. 
Carrión, F., Tarjuelo, J.M., Hernández, D., Moreno, M.A. (2013). Design of microirrigation subunit of minimum cost with proper operation. Irrig. Sci., 31(5), 1199-1211.

Karmeli, D., Keller, J. (1975). Trickle irrigation design. Rain Bird Sprinkler Manufacturing Corp. Glendora, 133p.

Moreno, M.A., Planells, P., Córcoles, J.I., Tarjuelo, J.M., Carrión, P.A. (2009). Development of a new methodology to obtain the characteristic pump curves that minimize the total cost at pumping stations. Biosyst. Eng., 102 (1), 95-105.

Moreno M.A., del Castillo A., Montero J., Tarjuelo J.M., Ballesteros R. (2016). Design optimization of irregular pressurized irrigation subunits. Biosyst. Eng. (en revision)

Moreno, M.A., Medina, D., Ortega, J.F., Tarjuelo, J.M. (2012). Optimal design of centre pivot systems with water supplied from wells. Agricultural Water Management, 107, 112-121.

Rossman, L.A. (2000). EPANET 2, users' manual, Water supply and Water Resources Division, National Risk Management Research Laboratory, U.S. Enviromental Protection Agency, Cincinnati. 